\title{
ADHESION, INTEGRATION AND OSTEOGENESIS OF HUMAN DENTAL PULP STEM CELLS ON BIOMIMETIC IMPLANT SURFACES COMBINED WITH PLASMA DERIVED PRODUCTS
}

\author{
I. Irastorza ${ }^{1}$, J. Luzuriaga ${ }^{1}$, R. Martinez-Conde ${ }^{2}$, G. Ibarretxe ${ }^{1}$ and F. Unda ${ }^{1 *}$ \\ ${ }^{1}$ Department of Cell Biology and Histology. Faculty of Medicine and Nursing, \\ University of the Basque Country, UPV/EHU, Leioa, 48940, Bizkaia, Spain. \\ ${ }^{2}$ Department of Stomatology II. Faculty of Medicine and Nursing, University of the Basque Country, \\ UPV/EHU, Leioa, 48940, Bizkaia, Spain.
}

\begin{abstract}
Dental implants are the usual therapy of choice in the dental clinic to replace a loss of natural teeth. Over recent decades there has been an important progress in the design and manufacturing of titanium implant surfaces with the goal of improving their osteointegration. In the present work, the aim was to evaluate the usefulness of hDPSCs (human dental pulp stem cells), in combination with autologous plasma components, for in vitro bone generation on biomimetic titanium dental implant materials. In this context, the combination of hDPSCs stimulated by PRGF or PRF and cultured on standard Ti6A14V and biomimetic BASTM (Avinent Implant System) titanium surfaces were studied in order to evaluate possible enhancements in the osteoblastic differentiation process out of human mesenchymal cells, as well as bone matrix secretion on the implant surface. The results obtained in this in vitro model of osteogenesis suggested a combination of biomimetic rough titanium surfaces, such as BAS ${ }^{\mathrm{TM}}$, with autologous plasma-derived fibrin-clot membranes such as PRF and/or insoluble PRGF formulations, but not with an addition of water-soluble supplements of plasma-derived growth factors, to maximise osteoblastic cell differentiation, bone generation, anchorage and osteointegration of titanium-made dental implants.
\end{abstract}

Keywords: Dental pulp stem cells, titanium implants, osteoblast differentiation, platelet rich in growth factors, platelet rich fibrin, biomimetic advanced surface.

*Address for correspondence: Fernando Unda, Cell Biology and Histology Department. Faculty of Medicine and Nursing, University of the Basque Country, UPV/EHU, Leioa, 48940, Bizkaia, Spain.

Telephone number: +34946012857 Email: fernandoundarodriguez@gmail.com

Copyright policy: This article is distributed in accordance with Creative Commons Attribution Licence (http://creativecommons.org/licenses/by-sa/4.0/).

\section{Introduction}

Dental implants are synthetic tooth-root replacement structures which are anchored to the alveolar bone to support different prosthetic suprastructures and thus recover the masticatory function of lost dental pieces. After half a century of the first successful trials with human patients (Albrektsson et al., 1981), dental implants are now well established as the usual therapy of choice in the dental clinic to replace a loss of natural teeth. The gold-standard material for intra-osseous dental implants is titanium or titanium alloy, due to its optimal resistance and durability. However, over recent decades there has been an important progress in the design and manufacturing of titanium implant surfaces with the goal of improving their osteointegration (Gasik et al., 2015; Rani et al., 2012; Rupp et al., 2018; Salou et al., 2015). For an effective anchorage of the titanium implant, it is important to consider not only its composition but also the topography of its surface (Annunziata and Guida, 2015; Jemat et al., 2015; Le Guéhennec et al., 2007; Naves et al., 2015). It has been demonstrated that rough titanium surfaces, which maximise the contact with the surrounding alveolar bone, present a better integration than smooth titanium ones (Coelho et al., 2009). This benefit can be at least partially attributed to the fact that surface microporosity enhances the osteoblastic differentiation of surrounding mesenchymal stem cells, which are ultimately responsible for bone healing and consolidation after the placement of the implant (Boyan et al., 2016; Graziano et al., 2008a). Thus, an activation of mesenchymal stem cells in the affected alveolar bone area is fundamental with a view to implant integration, to increase its resistance, durability, and eventually avoid rejection and/or possibly ensuing bacterial infections owing to an 
insufficient presence of bone to physically support masticatory impacts (e.g. Perimplantitis). In this context, the use of titanium implants, combined with cellular allografts/autografts, may offer substantial benefits for clinical implantology.

Human dental pulp stem cells (hDPSCs) from permanent teeth are ecto-mesenchymal stem cells with a neural crest origin (La Noce et al., 2014a). These cells differentiate very efficiently into boneproducing osteoblastic cells (Graziano et al., 2008b; Karbanová et al., 2010; Laino et al., 2006; Paino et al., 2017; Mortada and Mortada, 2018). When hDPSCs are seeded on to the polystyrene plastic surfaces usually found in manufactured plates for in vitro culture, they need the addition of osteogenic differentiation factors to give rise to bone-producing osteoblasts. A typical osteoblastic differentiation recipe contains dexamethasone, $\beta$-glycerophosphate and ascorbate (Aurrekoetxea et al., 2015; Langenbach and Handschel, 2013). Despite their obvious osteogenic characteristics, hDPSCs have only had a relatively limited application in implantology research, with few, albeit successful, clinical trials performed so far (Giuliani et al., 2013; La Noce et al., 2014b). However, in some comparative studies with other mesenchymal stem cells such as bone marrow stem cells, hDPSCs have proven to be the most effective in promoting in vivo osteointegration of titanium implants (Ito et al., 2011; Mangano et al., 2010). Clinical implantology could still be missing a "gold mine" for the development of personalised therapies with a view to improving dental implant integration, since the lost tooth could also eventually make a contribution to subsequent implant success, by providing a highly valuable source of autologous hDPSCs.

Plasma rich in growth factors (PRGF) and platelet rich fibrin (PRF) are plasma-derived advanced medical therapy products (AMTP) (Giannini et al., 2015; Kobayashi et al., 2016; Nishiyama et al., 2016) whose usage is currently much extended in the dental clinic to promote bone healing following tooth extraction, and preparation for a subsequent titanium pillar placement. Both formulations contain a complex mixture of factors that induce proliferation and differentiation to osteoblastic cells. They also have the great advantage that they can be used in autologous personalised therapies (Anitua et al., 2013). These technologies are currently used to induce tissue regeneration at both the experimental and clinical level, but especially in implant dentistry therapies (Anitua et al., 2016; Masuki et al., 2016).

PRGF consists of an autologous platelet-enriched plasma fraction obtained from centrifugation of a patient's blood sample, which is then activated by calcium chloride. This causes massive platelet degranulation, and leads eventually to the formation of a clot containing polymerised fibrin and a complex mixture of growth factors with regenerative potential (Anitua et al., 2009; Jovani-Sancho et al., 2016; Paknejad et al., 2012). These factors may be subsequently separated from the fibrin-clot as a soluble fraction following centrifugation (Anitua et al., 2012).

PRF is obtained by fast centrifugation of fresh blood in the absence of anticoagulants, and contains autologous leukocytes and platelets embedded in a fibrin clot, which can be physically manipulated and flattened into thin membranes that progressively release growth factors upon cell degranulation over time. The presence of growth factors and cytokines that derive from blood platelets and leukocytes stimulate fast tissue healing during the first days following surgery. PRF promotes cell chemotaxis and proliferation, and the resulting fibrin containing matrix is resistant, strong, flexible and easy to manipulate, being adaptable to different anatomical surfaces (Khurana et al., 2017; Kumar et al., 2016). The work described suggests that a combination of a highly osteogenic and autologous cell source, such as hDPSCs, together with microporous titanium structures and plasma-derived products could provide an ideal strategy with a view to enhancing current implant therapies.

In the current study, the osteogenesis of hDPSCs seeded in vitro over titanium discs (Ti6Al4V), with polished surfaces either non-treated or pretreated with a BAS ${ }^{\mathrm{TM}}$ (Biomimetic Advanced Surface; Avinent Implant System) protocol, was assessed. The BAS surface was obtained by using aluminium oxide and then anodised by an electrolyte solution. This treatment generates the effect of macroroughness and microporosity on the titanium surface (Santander et al., 2012). Despite the proven ability of biomimetic BAS $^{\text {TM }}$ titanium implants for osteo-integration, it was not known whether BAS ${ }^{\mathrm{TM}}$ surfaces would improve osteogenesis from hDPSCs and whether this bone deposition could then be, in turn, enhanced or not by the presence of PRGF/PRF. To answer this question, the different steps of the osteoblastic differentiation process from hDPSCs were analysed by using stagespecific genetic and phenotypic biomarkers, assessing how each of these were affected by the combined presence of BAS ${ }^{\mathrm{TM}}$ surfaces and plasma-derived products with different physical (PRGF soluble vs. PRF non-soluble) formulations.

\section{Materials and Methods}

\section{Titanium disc preparation}

Ti6Al4V ELI $2 \mathrm{~mm}$ thick discs were cut from a $5.5 \mathrm{~mm}$-diameter bar, provided by Avinent Implant System SLU (Barcelona, Spain). To prepare the BAS ${ }^{\text {TM }}$ (Biomimetic Advanced Surface) surface, one of the disc faces was treated by shot blasting using white corundum $\left(\mathrm{Al}_{2} \mathrm{O}_{3}\right) \mathrm{F} 60$ with projection particle sizes of 212-300 $\mu \mathrm{m}$. The shot blast discs were then washed in an ultrasonic bath for $10 \mathrm{~min}$ and rinsed with deionised water. Next, the discs were anodised by connecting them to the anode of a DC power source and using an aqueous solution rich in calcium and 
phosphorus as an electrolyte. A current density of $0.75 \mathrm{~mA} / \mathrm{mm}^{2}$ was applied and the potential was allowed to increase freely until it reached the value of $130 \mathrm{~V}$. Subsequently, the samples were rinsed with deionised water in an ultrasonic bath for $10 \mathrm{~min}$. The control discs Ti6Al4V were machined without any surface treatment or polishing.

\section{hDPSC isolation and culture}

Human pulp stem cells (hDPSCs) were obtained from the third molar teeth of young healthy patients (age 18-30) in dental clinics, with the informed consent of the patient. The procedures required have been officially approved by the competent authority (Ethics committee of the University of the Basque Country) under the M10_2016_088 protocol.

Dental pulp was extracted by molar fracture and digested using an enzymatic solution containing $3 \mathrm{mg} / \mathrm{mL}$ collagenase type I (Gibco, NY, USA) and $4 \mathrm{mg} / \mathrm{mL}$ dispase (Sigma, MO, USA) in Hank's balanced salt solution (HBSS, Gibco, NY, USA), and then incubated for $1 \mathrm{~h}$ at $37^{\circ} \mathrm{C}$. The enzymatic solution was neutralised using Dulbecco's modified eagle medium (DMEM, Lonza, Switzerland) supplemented with $10 \%$ foetal bovine serum (FBS, HyClone, USA), and centrifuged at $280 \times g$ for $5 \mathrm{~min}$. The obtained pellet was physically dissociated using a syringe with an $18 \mathrm{G}$ needle. After disruption, the cells were seeded in a $25 \mathrm{~cm}^{2}$ flask containing DMEM and $10 \%$ FBS, $1 \%$ penicillin-streptomycin (Gibco, NY, USA) and $1 \%$ L-glutamine (Sigma, MO, USA) at $37^{\circ} \mathrm{C}$ in $5 \% \mathrm{CO}_{2}$. Dental pulp cells were then subcultured to $75 \mathrm{~cm}^{2}$ flasks (Sarstedt, Nümbrecht, Germany), when they reached $80 \%$ confluence. Cell cultures between 4-10 passages and a maximum age of four months were used for the experiments.

\section{Culture of hDPSCs on titanium discs}

hDPSCs were cultured until $80 \%$ confluence and then detached from the culture flasks by using trypsinethylenediaminetetraacetic acid (EDTA) at $20 \%$ for $5 \mathrm{~min}$ at $37^{\circ} \mathrm{C}$. After counting, cells were seeded at different densities for different assays on Ti6Al4V and BAS $^{\mathrm{TM}}$ titanium discs. To enhance cell attachment the seeding was performed by placing a drop of $70 \mu \mathrm{L}$ $\left(15,000\right.$ cells) on the disc and incubating at $37^{\circ} \mathrm{C}$ for $3 \mathrm{~h}$. After the incubation, the discs were covered with $700 \mu \mathrm{L}$ of supplemented DMEM medium on 24-well plates or $4 \mathrm{~mL}$ of medium on 6-well plates. To perform the hDPSC culture experiments, 6 discs were used per condition and the experiments were carried out in triplicate.

\section{Flow cytometry}

The hDPSC phenotype was evaluated by flowcytometry analysis. Fluorophore-labelled rabbit antihuman antibodies for the following surface antigens were used: CD105-PE, CD90-FITC, CD73-APC and CD45-APC (Thermo Fisher Scientific, MA, USA). Cultured cells were detached from the flasks and washed with phosphate-buffered saline (PBS) and then with PBS containing $2 \%$ Bovine serum albumin (BSA, Sigma, MO, USA) and centrifuged at $180 \times g$ for $5 \mathrm{~min}$. The hDPSCs were then resuspended into $100 \mu \mathrm{L}$ of PBS with $2 \%$ BSA and incubated with the aforementioned antibodies in the dark on ice, for $50 \mathrm{~min}$. After the incubation, the cells were washed with PBS with $2 \%$ BSA and centrifuged at $180 \times g$ for $5 \mathrm{~min}$ at room temperature to remove residual antibodies. The pellet with the cells was resuspended in $500 \mu \mathrm{L}$ of PBS. Cytometry analysis was performed using a Gallios flow cytometer (Beckman Coulter, CA, USA) and data were analysed using Kaluza 1.1 software (Beckman Coulter, CA, USA).

\section{Calcein/propidium iodide staining}

hDPSCs cultured for $4 \mathrm{~d}$ on titanium discs were washed with PBS. The cells were incubated with $3 \mu M$ calcein-AM (Molecular probes, OR, USA) and $2.5 \mu \mathrm{M}$ propidium iodide (Sigma, MO, USA) for $30 \mathrm{~min}$ at $37^{\circ} \mathrm{C}$. After incubation, the cells were washed three times with PBS. hDPSCs on discs were observed and images captured using an Axioskop fluorescence microscope (Zeiss, Overkochen, Germany) and a Nikon DS-Qi1 camera (Nikon, Tokyo, Japan).

\section{Scanning electron microscopy}

hDPSCs cultured on Ti6Al4V and BAS discs were fixed in $2 \%$ glutaraldehyde (Sigma, MO, USA) in $0.1 \mathrm{M}$ Sorensen phosphate buffer (SPB, Thermo Fisher Scientific, MA, USA) for $1 \mathrm{~h}$ at $4{ }^{\circ} \mathrm{C}$. The cells were washed 3 times with $4-8 \%$ sucrose in $0.1 \mathrm{M}$ $\mathrm{SPB}$ and then another 3 times with $0.1 \mathrm{M}$ SPB. The samples were dehydrated by a series of $15 \mathrm{~min}$ washes of ethanol $(30 \%, 50 \%, 70 \%, 90 \%$, and $100 \%$ ) (Panreac, Barcelona, Spain) and a final wash in hexamethyldisilazane (Sigma, MO, USA). The samples were air-dried after dehydration and sputter coated with $15 \mathrm{~nm}$ of gold. Samples were examined using a scanning electron microscope (SEM) (S4800, Hitachi High Technologies, Tokyo, Japan). Titanium discs without cells were also studied by SEM.

\section{Preparation of PRGF and PRF, and culture on hDPSCs}

To obtain plasma rich in growth factors (PRGF), blood was collected from a healthy young donor (male, 26 years old) in $5 \mathrm{~mL}$ blood collection tubes (BD Vacutainer, Plymouth, UK) with $3.8 \%$ sodium citrate anticoagulant. The tubes were centrifuged at $580 \times g$ for $8 \mathrm{~min}$ at room temperature. The Plateletrich fraction was transferred to a $15 \mathrm{~mL}$ falcon tube and activated by $10 \%$ calcium chloride $(50 \mu \mathrm{L}$ in $1 \mathrm{~mL}$ plasma) for $1 \mathrm{~h}$ at $37^{\circ} \mathrm{C}$. Following filtration, the plasma was then centrifuged at $3000 \times g$ for $15 \mathrm{~min}$ at $4{ }^{\circ} \mathrm{C}$. Plasma soluble fractions were aliquoted and stored at $-20^{\circ} \mathrm{C}$, until their use in hDPSC cultures at $20 \%$ volume. Fresh PRGF was added to the cultures with every change of medium every $48 \mathrm{~h}$.

To obtain platelet rich fibrin (PRF), blood was collected as previously described but using collection tubes without anticoagulant (BD Vacutainer, 
Plymouth, UK). The samples were centrifuged at $580 \times g$ for $8 \mathrm{~min}$ at room temperature. The plateletcontaining fibrin clot was separated from the haematocrit using forceps and scalpel and flattened between two glass surfaces by weight application (552 Pa) for $1 \mathrm{~h}$ at room temperature. The obtained fibrin clot membranes were cut in to smaller sections of around $1 \mathrm{~cm}^{2}$, and immediately placed upon the hDPSC cultures. PRF membranes were kept in the culture wells independently of periodic $(48 \mathrm{~h})$ medium changes, for the whole duration of the experiment.

\section{Immunocytochemistry}

After $4 \mathrm{~d}$ of culture on titanium discs, hDPSCs were washed with PBS and fixed in $4 \%$ paraformaldehyde (PFA) for $10 \mathrm{~min}$ at room temperature. The cells were then incubated with $10 \%$ goat serum (Invitrogen, CA, USA) for $10 \mathrm{~min}$ at room temperature, followed by overnight incubation at $4{ }^{\circ} \mathrm{C}$ with either Ki67 primary antibody (Rabbit polyclonal, Abcam, Cambridge, UK) or collagen type I primary antibody (Rabbit polyclonal, Abcam, Cambridge, UK) at 1:500 dilution using $0.1 \%$ Triton X-100/1 \%BSA/PBS. The secondary antibody, alexa fluor 488 conjugated rabbit IgG (Abcam, Cambridge, UK), was used for primary antibody localisation for $1 \mathrm{~h}$ at room temperature. Nuclei were stained with 4',6-diamino-2-fenilindol (DAPI, Invitrogen, CA, USA). The images were taken using an Axioskop fluorescence microscope (Zeiss, Overkochen, Germany) and Nikon DS-Qi1 camera (Nikon, Tokyo, Japan).

\section{Osteogenic differentiation of hDPSCs}

In some experiments, hDPSCs were cultured with osteogenic medium for osteodifferentiation. The DMEM medium was supplemented with $50 \mu \mathrm{M}$ ascorbic acid (Sigma, MO, USA), $20 \mathrm{mM}$ $\beta$-glycerophosphate (Sigma, MO, USA) and $10 \mathrm{nM}$ dexamethasone (Sigma, MO, USA) for $14 \mathrm{~d}$ and in the presence or absence (control) of PRGF and PRF.

\footnotetext{
Alkaline phosphatase (ALP) staining

After culturing hDPSCs for $14 \mathrm{~d}$ in the presence or absence of osteogenic medium, cells were fixed briefly using $4 \%$ PFA for $1 \mathrm{~min}$ and washed with $0.05 \%$ Tween 20-PBS. ALP staining was performed using 5-bromo-4-chloro-3-indolyl phosphate/nitro blue tetrazolium (NBT/BCIP; Sigma, MO, USA) as a substrate, and the staining progress was checked every $3 \mathrm{~min}$. After the incubation, the cells were washed with PBS three times for $5 \mathrm{~min}$. The images were acquired using a Zeiss Stemi 2000-C stereoscopic microscope (Zeiss, Germany) and Canon PowerShot A80 camera (Canon, Tokyo, Japan). For quantification of ALP enzymatic activity, the cells were detached from the titanium surfaces using Trypsin-EDTA, and resuspended in a NBT/BCIP solution into a 24well culture plate. The absorbance was measured at $420 \mathrm{~nm}$ using a Synergy HT microplate reader
}

(BioTek, VT, USA) equipped with the Gen5 1.11 program.

\section{Alizarin red staining (ARS)}

After $14 \mathrm{~d}$ of culture, hDPSCs cultured with or without osteogenic medium were fixed with $4 \%$ PFA for $10 \mathrm{~min}$. The cells were then washed with distilled water and stained for $45 \mathrm{~min}$, in the dark at room temperature, using $2 \%$ ARS (Acros organics, Sigma, MO, USA) at $\mathrm{pH}$ 4.1-4.3. The staining solution was then washed off with distilled water until no ARS could be observed dissolved in the water. The images of stained bone precipitates were taken using a Zeiss Stemi 2000-C stereoscopic microscope (Zeiss, Germany) and a Canon PowerShot A80 camera (Canon, Tokyo, Japan). Quantification of bone matrix deposition was performed by dissolving the alizarinred-stained calcified deposits with acetic acid and measuring the absorbance at $405 \mathrm{~nm}$ using a Synergy HT microplate reader (BioTek, VT, USA).

\section{Quantitative real-time PCR analysis}

Cells were seeded on a density of $10^{5}$ cells per disc, and 5 discs were placed together on each well of a 6-well plate. After culturing hDPSCs for $14 \mathrm{~d}$, total RNA was extracted from the cells using a RNeasyPlus Mini Kit (Quiagen, Hilden, Germany). RNA purity and concentration were calculated using a Synergy HT spectrophotometer (Winooski, Vermont, USA) at 260/280 nm. Reverse transcription reaction was performed using an iScript ${ }^{\mathrm{TM}}$ cDNA synthesis Kit (BIO-RAD, Hercules, California, USA). The conventional polymerase chain reaction (PCR) was performed by MyTaq ${ }^{\mathrm{TM}}$ HS Red Mix, 2× (Bioline, London, UK). The amplification program was based on a first step at $95^{\circ} \mathrm{C}$ for $1 \mathrm{~min}$, followed by 30 cycles of $95^{\circ} \mathrm{C}$ for $15 \mathrm{~s}, 59^{\circ} \mathrm{C}$ for $15 \mathrm{~s}$ and $72{ }^{\circ} \mathrm{C}$ for $10 \mathrm{~s}$. Data were collected using a high-performance-ultraviolet transilluminator (UVP, Analytic Jena, Cambridge, UK).

The Q-PCR was performed using a Power SYBR Green PCR Master Mix (Applied biosystems, CA, USA). The amplification program consisted of an initial step at $95{ }^{\circ} \mathrm{C}$ for $10 \mathrm{~min}$, followed by 40 cycles of $95^{\circ} \mathrm{C}$ for $20 \mathrm{~s}$ and $59^{\circ} \mathrm{C}$ for $1 \mathrm{~min}$. $\beta$-actin and Gapdh were used as housekeeping genes and the four targeted genes were, collagen I (COL I), osteonectin (SPARC), runx2 (RUNX2) and osterix $(O S X)$. Sequences of forward and reverse primers were as follows: 5'-GTTGTCGACGACGAGCG-3' and 5'-GCACAGAGCCTCGCCTT-3' for $\beta$-ACTIN; 5' CTTTTGCGTCGCCAG $-3^{\prime}$ and 5'TTGATGGCAACAATATCCAC -3' for GAPDH; 5'-GGCCCCCTGGTATGACTGGCT-3' and 5'-CGCCACGGGGACCACGAATC-3' for COL I; 5'-GAAAGAAGATCCAGGCCCTC-3' and 5'-CTTCAGACTGCCCGGAGA-3' for SPARC; 5' - CACTCACTACCACACCTACC-3' and 5'-TTCCATCAGCGTCAACAC-3' for RUNX2; 5' - TGAGGAGGAAGTTCACTATG-3' and 
5'-CATTAGTGCTTGTAAAGGGG-3' for OSX. The expressions of target genes were normalised against the housekeeping gene.

\section{Statistical analysis}

The experimental data were statistically analysed using SPSS version 22.0 (IBM). Statistical comparisons were performed by ANOVA with post-hoc tests and confidence intervals were fixed at $95 \%(p<0.05), 99 \%$ $(p<0.01)$ and $99.9 \%(p<0.001)$.

\section{Results}

hDPSC isolation, culture and characterisation by flow cytometry

Isolated human dental pulp stem cells (hDPSCs) from impacted third molars were cultured in DMEM containing foetal bovine serum $(10 \%)$. Cells formed multiple colonies and, after 2-3 weeks, adherent hDPSCs reached subconfluence. Morphologically, the cells displayed a spindle-like fibroblast shape, and cultured cells maintained this morphology as well as a high proliferation rate after several passages (Fig. 1a-d).

To evaluate the marker expression profile of the cultured cells, hDPSCs were analysed by flow cytometry using antibodies for the following CD (clusters of differentiation) antigens: CD105, CD90, CD73 and CD45. hDPSCs were positive for mesenchymal stem cell markers CD105 (100\%), CD73 (100 \%) and CD90 (100\%), but negative for the haematopoietic markerCD45. These results demonstrated the mesenchymal stem cell phenotype of the dental pulp isolated cells (Fig. 1e-h).

\section{Microtopographical analysis of $\mathrm{Ti6414V}$ and} BAS $^{\mathrm{TM}}$ titanium surfaces and cell adhesion by scanning electron microscopy

The SEM images of the original and cell-free raw titanium material surface (Ti6A14V; Grade 5 ELI Ti) displayed concentrically parallel grooves (Fig. 2a), whereas the rough surface (BAS ${ }^{\mathrm{TM}}$ ) had a structure with numerous pits and bumps and many very small $(<10 \mu \mathrm{m})$ micropores dispersed uniformly throughout the whole sample surface (Fig. 2c).

\section{Cell adhesion and migration of hDPSCs on Ti6A14V and BAS ${ }^{\mathrm{TM}}$ titanium surfaces}

20,000 hDPSCs were placed in culture on each of the titanium samples for $4 \mathrm{~d}$. The cells strongly adhered to and spread out on the smooth surface of the Ti6A14V titanium, following the lines of the disc's groove pattern and showing numerous cell elongations similar to lamellipodia (Fig. 2b). However, on the BAS surfaces, hDPSC cells adhered to the porous surface by adapting to the surface contour morphology, and cells penetrated deeper into the porous structure (Fig. 2d). In both cases, cell adhesions appeared to be very consistent and maintained throughout the full time of culture.

\section{Cell viability of hDPSCs on Ti6A14V and BAS ${ }^{\mathrm{TM}}$ titanium surfaces}

hDPSCs were cultured for $4 \mathrm{~d}$ on titanium samples in the presence of Calcein-AM (green fluorescence) and propidium iodide (red fluorescence), to study cell viability and cell death, respectively. hDPSCs were $100 \%$ calcein positive on both smooth and rough surfaces, which demonstrated the high viability of these cells on the titanium discs (Fig. 2e-g). Moreover,
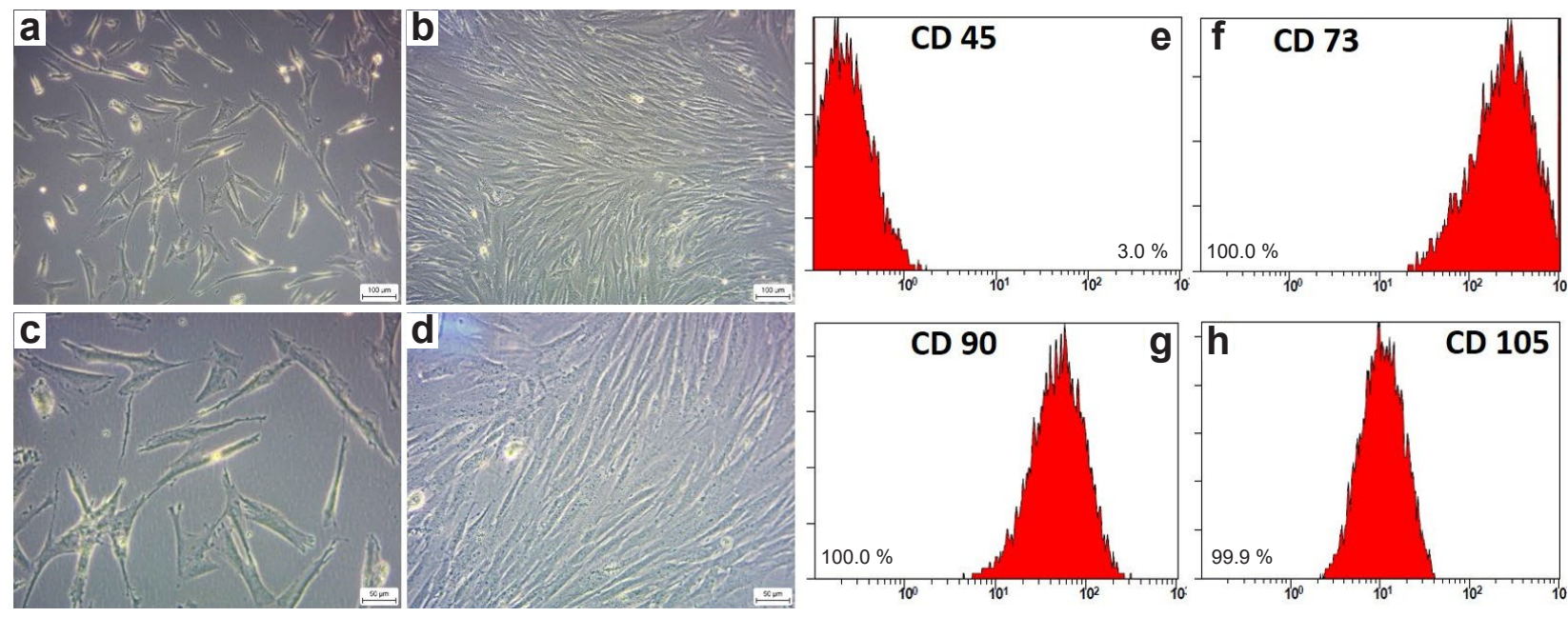

Fig. 1. Culture of hDPSCs and stem cell markers by flow cytometry. a-d: Phase-contrast microscopy images for adherent hDPSCs in culture under subconfluence $(\mathbf{a}, \mathbf{c})$ and confluence $(\mathbf{b}, \mathbf{d})$ at low and high magnification. Flow cytometry revealed that hDPSCs were all negative for the haematopoietic marker CD45 (e) and all positive for the mesenchymal stem cell markers CD73, CD90 and CD105 (f,g,h, respectively). 


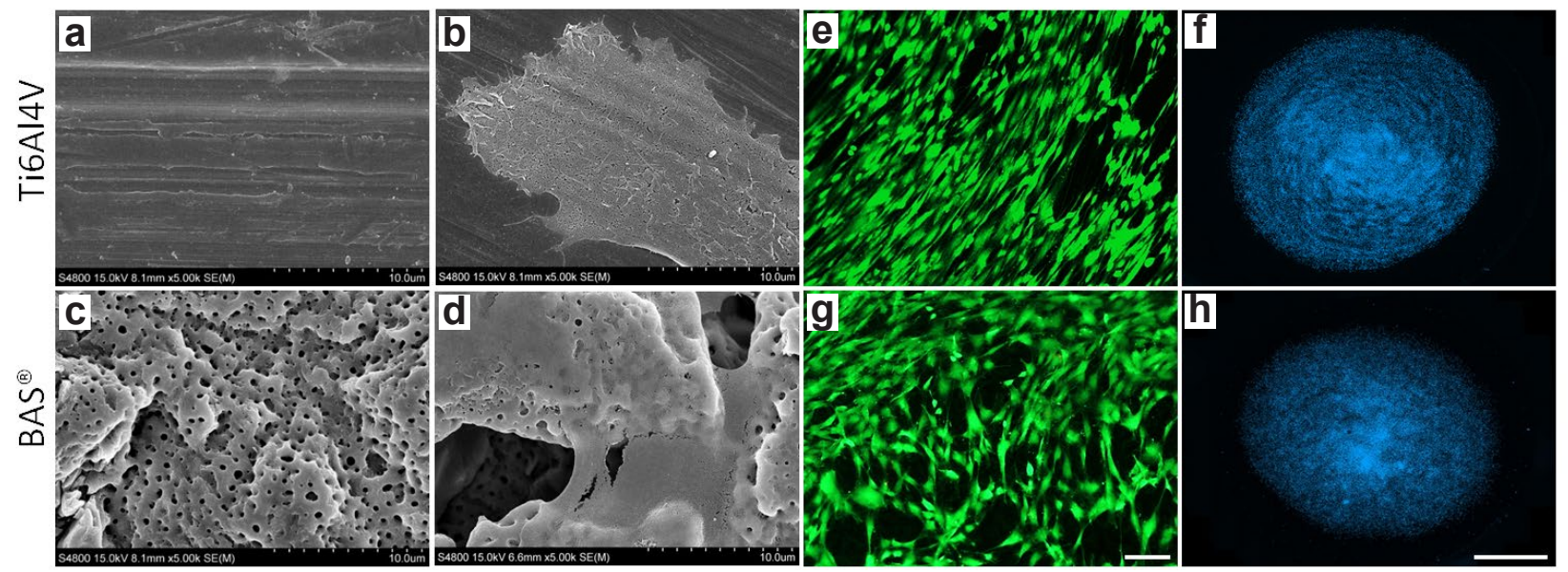

Fig. 2. SEM images and viability of hDPSCs on titanium surfaces. Scanning electron microscopy images of titanium surfaces, Ti6Al4V (a) and BAS (c), and hDPSC cells seeded onto both surfaces (b,d, respectively) Fluorescent microscopy of hDPSCs cultured for $4 \mathrm{~d}$ on titanium discs and then tested for cell viability by calcein (green) and for cell death by propidium iodide (red) staining (e,g). Scale bar: 100 $\mu$ m. Images of entire discs with hDPSC nuclei stained in blue with DAPI on Ti6Al4V (f) titanium surface and BAS samples (h). Scale bar: $2 \mathrm{~mm}$.
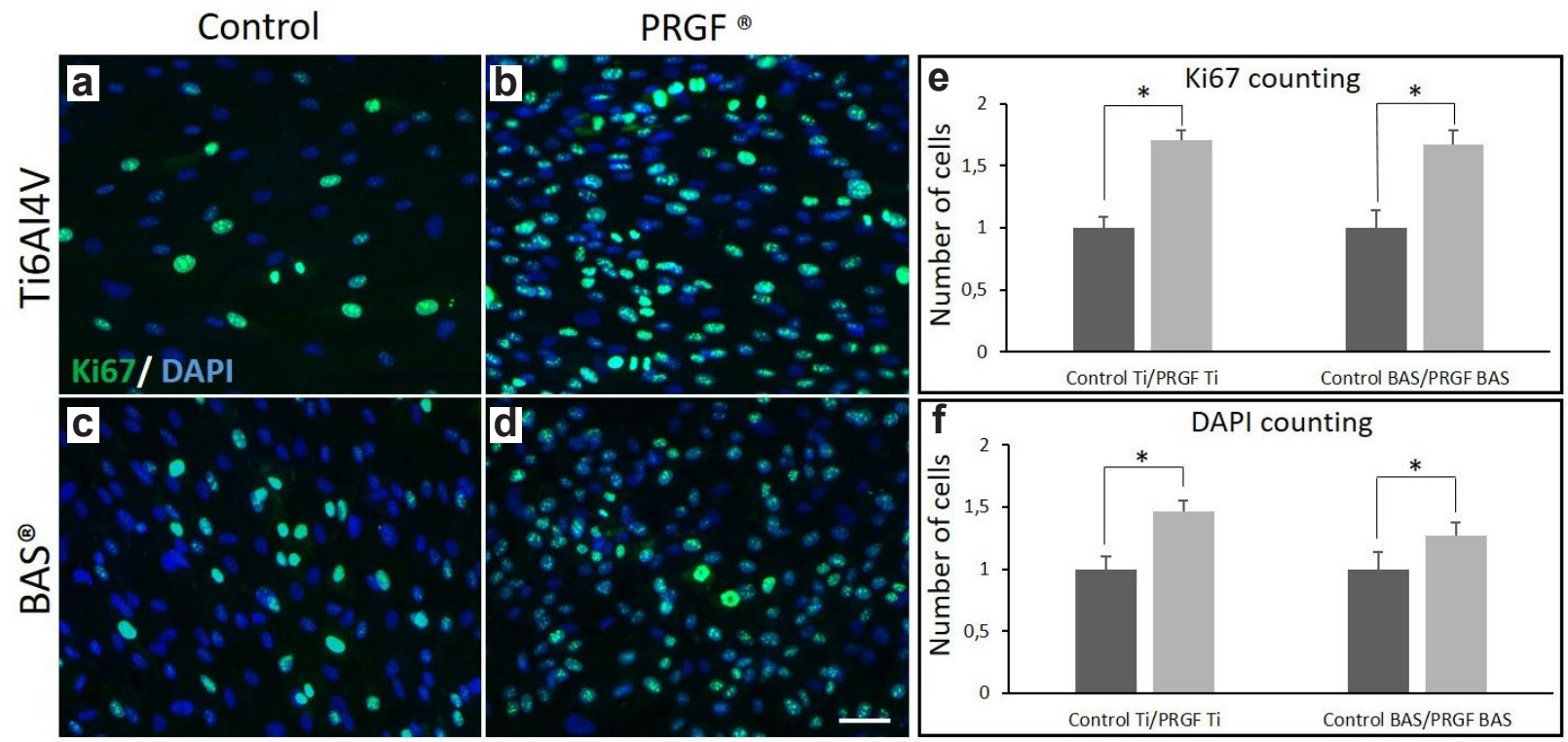

Fig. 3. Proliferation assay of hDPSCs cultured on titanium surfaces. Immunofluorescence of hDPSCs for Ki67 protein (green) and counterstained by DAPI (blue) in control conditions on Ti6Al4V (a) and BAS (c) discs, and cultured with $20 \%$ PRGF on Ti6Al4V (b) and BAS (d) discs, for $4 \mathrm{~d}$. Cell proliferation increased under the PRGF treatment. Scale bar: $50 \mu \mathrm{m}$. Graphs illustrating comparison between control (normalised number of cells) and PRGF conditions in the total amount of cycling cells (Ki67+; e) and number of nuclei (DAPI; f) of cells grown on both titanium surfaces. Statistical significance was set at $p \leq 0.05$.

red fluorescence due to propidium iodide was not detected in any of the hDPSCs on titanium surfaces, which showed that levels of cell death was negligible in these cultures.

Images of entire titanium discs, with hDPSC nuclei stained in blue by DAPI, revealed a spiral orientation of the cells following the groove directions of the Ti6Al4V surfaces (Fig. 2f), whereas no preferential orientation of cells was observed in the BAS samples (Fig. 2h).
Cell proliferation assay of hDPSCs on Ti6A14V and BAS ${ }^{\mathrm{TM}}$ titanium surfaces combined with PRGF

Given that opaque titanium surfaces precluded a precise photometric/fluorometric quantification of the viable proliferating cells, the total numbers of viable cells (interphase or mitosis) on the discs were counted, together with assessing their nuclear morphology by using the DNA fluorescent stain DAPI. It was found that nuclear morphology was 


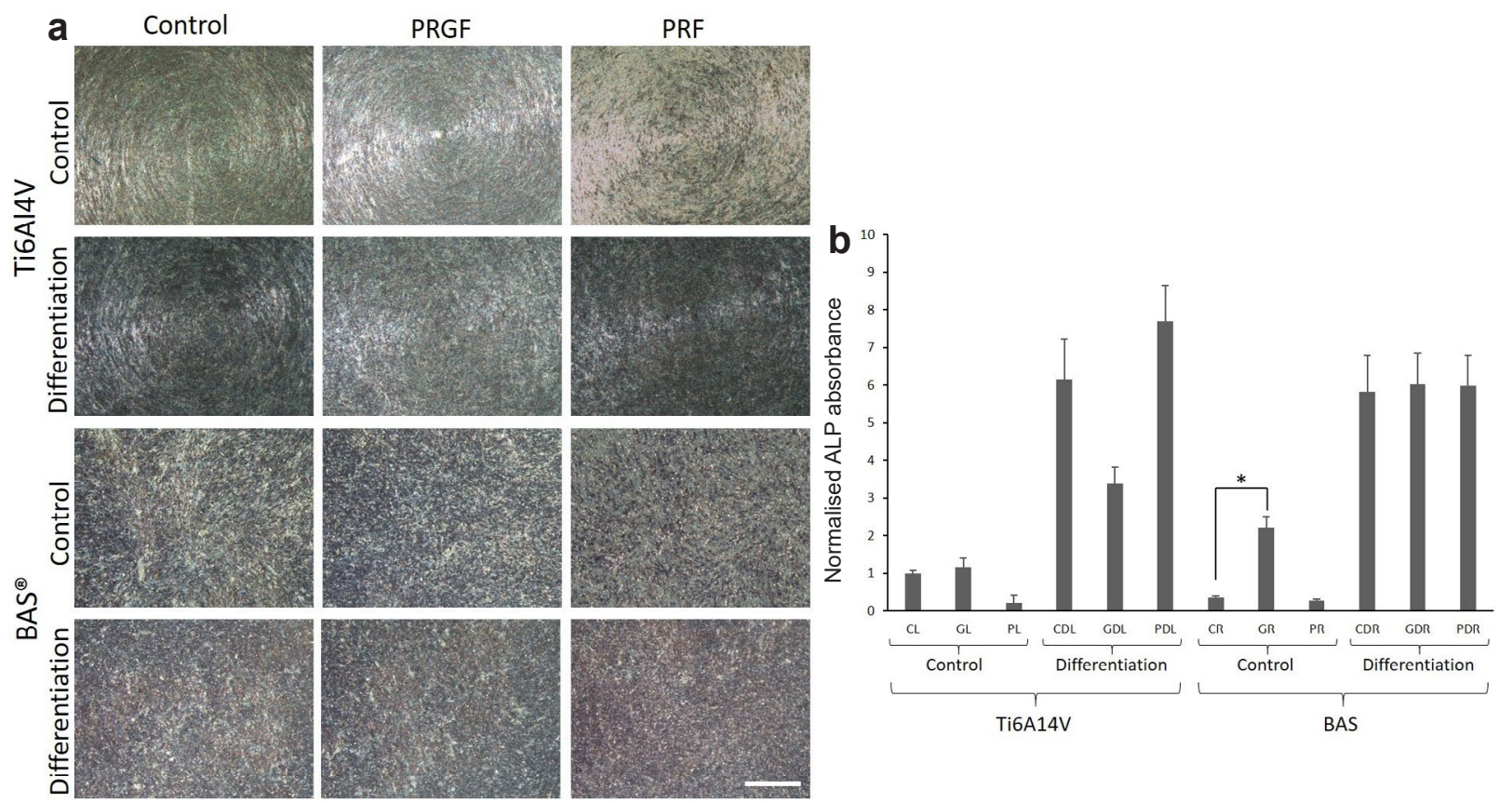

Fig. 4. Alkaline phosphatase assay of hDPSCs cultured on titanium surfaces. Alkaline phosphatase staining of hDPSC cultured for $14 \mathrm{~d}$ on Ti6Al4V and BAS titanium discs (a) with differentiation media, PRF and PRGF. Normalised quantification of the ALP activity $(\mathbf{b})$. Statistical significance was set at $\left({ }^{*}\right) p \leq 0.05$. Scale bar: $1 \mathrm{~mm}$.

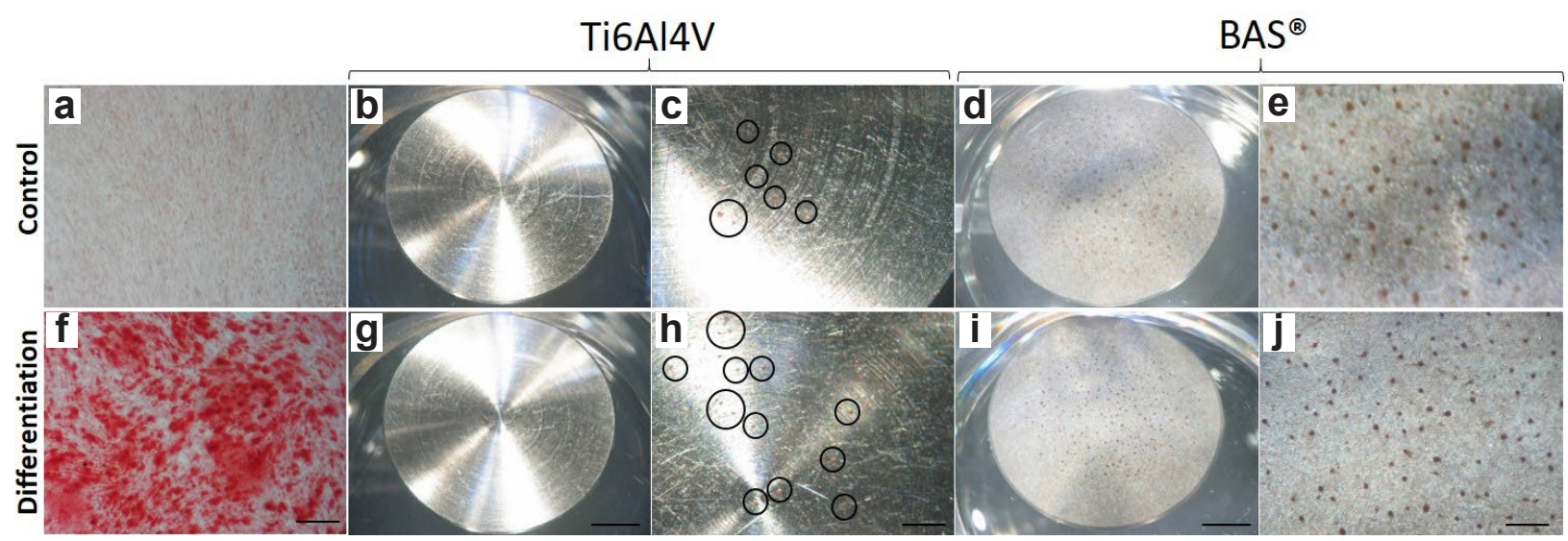

Fig. 5. Alizarin red assay of hDPSCs cultured on titanium surfaces. ARS of hDPSCs cultured for $14 \mathrm{~d}$ in control conditions on plastic (a), Ti6Al4V (b-c) and BAS (d-e) titanium discs in comparison with cells in osteoblastic differentiation media on plastic (f), Ti6Al4V (g-h) and BAS (i-j). Scale bars (a,f): $100 \mu \mathrm{m}$; Scale bars $(\mathbf{b}, \mathbf{g}, \mathbf{d}, \mathbf{i}): 2 \mathrm{~mm}$; Scale bars $(\mathbf{c}, \mathbf{h}, \mathbf{e}, \mathbf{j}): 0.5 \mathrm{~mm}$.

regular and even for interphase cells, and condensed chromosomes were visible in mitotic cells. As expected, most hDPSCs appeared to be in interphase, although some were at different stages of mitosis, mainly in metaphase and early and late anaphase, which indicated that the hDPSCs had grown and proliferated normally. However, when hDPSCs were cultured in the presence of soluble PRGF, significantly higher $(20$ to $50 \%$; $p<0.005)$ nuclear cell counts were consistently obtained after $4 \mathrm{~d}$ with respect to control conditions (Fig. 3). This indicated that the presence of plasma-derived supplements induced an increase in proliferation of hDPSCs grown on titanium surfaces. A Ki67 immunoassay was used to corroborate these findings. Again, a significant increase in Ki67 positive cells was found when hDPSCs were cultured in the presence of PRGF on either Ti6A14V or BAS titanium surface disc samples. (Fig. 3a-e).

\section{Alkaline phosphatase activity and osteogenic commitment of hDPSCs seeded on Ti6A14V and BAS $^{\text {TM }}$ titanium surfaces}

Stem cell differentiation into osteoblasts is typically assessed by examining the levels of ALP activity, an early osteoblast marker which is necessary for the onset of extracellular matrix mineralisation (Fig. 4a-b). In order to maximise osteoblastic differentiation, a well-known treatment consisting of the addition of dexamethasone, ascorbic acid and $\beta$-glycerophosphate to the culture medium for 

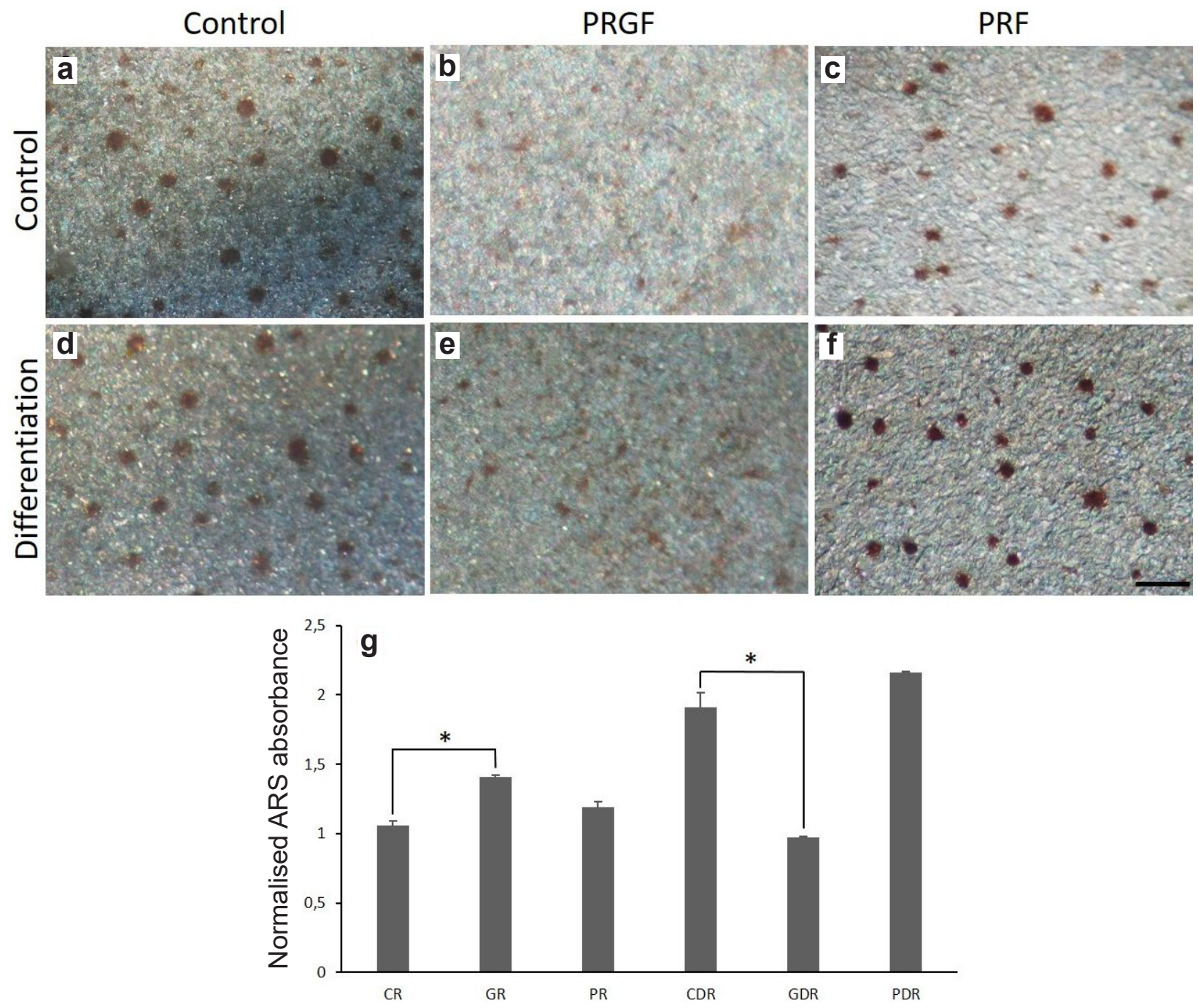

Fig. 6. Alizarin red staining of hDPSCs cultured with plasma derived supplements on BAS titanium surface. ARS of hDPSCs cultured for $14 \mathrm{~d}$ (a) on BAS titanium discs in control or differentiation treatment in the absence or presence of PRGF and PRF. Normalised quantification of ARS (b). Statistical significance was set at $\left(^{*}\right) p \leq 0.05$. Scale bar: $250 \mu \mathrm{m}$.

$7 \mathrm{~d}$, was applied to the Ti6A14V and BAS-seeded hDPSCs. In addition, PRGF and PRF were added to some cultures. Levels of ALP activity markedly increased when hDPSCs were treated with the osteoblastic differentiation reagents, both in smooth and rough titanium surface cultures. Additionally, in differentiation treatments, PRGF did not increase ALP enzyme activity, which was consistent with the higher proliferation rates of the cells cultured in the presence of PRGF (Fig. 3). However, the addition of non-soluble PRF increased ALP activity in cells on smooth titanium samples, with respect to differentiation controls. Curiously, in the absence of differentiation treatment, PRGF also increased ALP activity in the wells containing cells growing on BAS surfaces. This was probably due to the existing higher cell density, caused by the induction of cell proliferation (Fig. 3b).

\section{ARS and terminal osteogenic differentiation of hDPSCs seeded on Ti6A14V and BAS ${ }^{\mathrm{TM}}$ titanium surfaces}

The formation of calcified bone matrix nodules is the ultimate evidence of terminal osteogenic differentiation. This was assessed in hDPSC cultures by ARS, which stains calcified extracellular bone matrix in orange/red. To assess osteogenic activity of hDPSCs grown on different plastic/titanium surfaces, cells were seeded on either Ti6A14V or BAS surfaces and treated for $14 \mathrm{~d}$ with osteoblastic differentiation reagents, and cell cultures were then stained with alizarin red.

Stereoscopic microscope images showed no ARS whatsoever when hDPSCs where seeded on plastic. In contrast, when hDPSCs were cultured on titanium surfaces, especially on BAS surfaces, unmistakable red spots corresponding to calcified matrix accumulations 

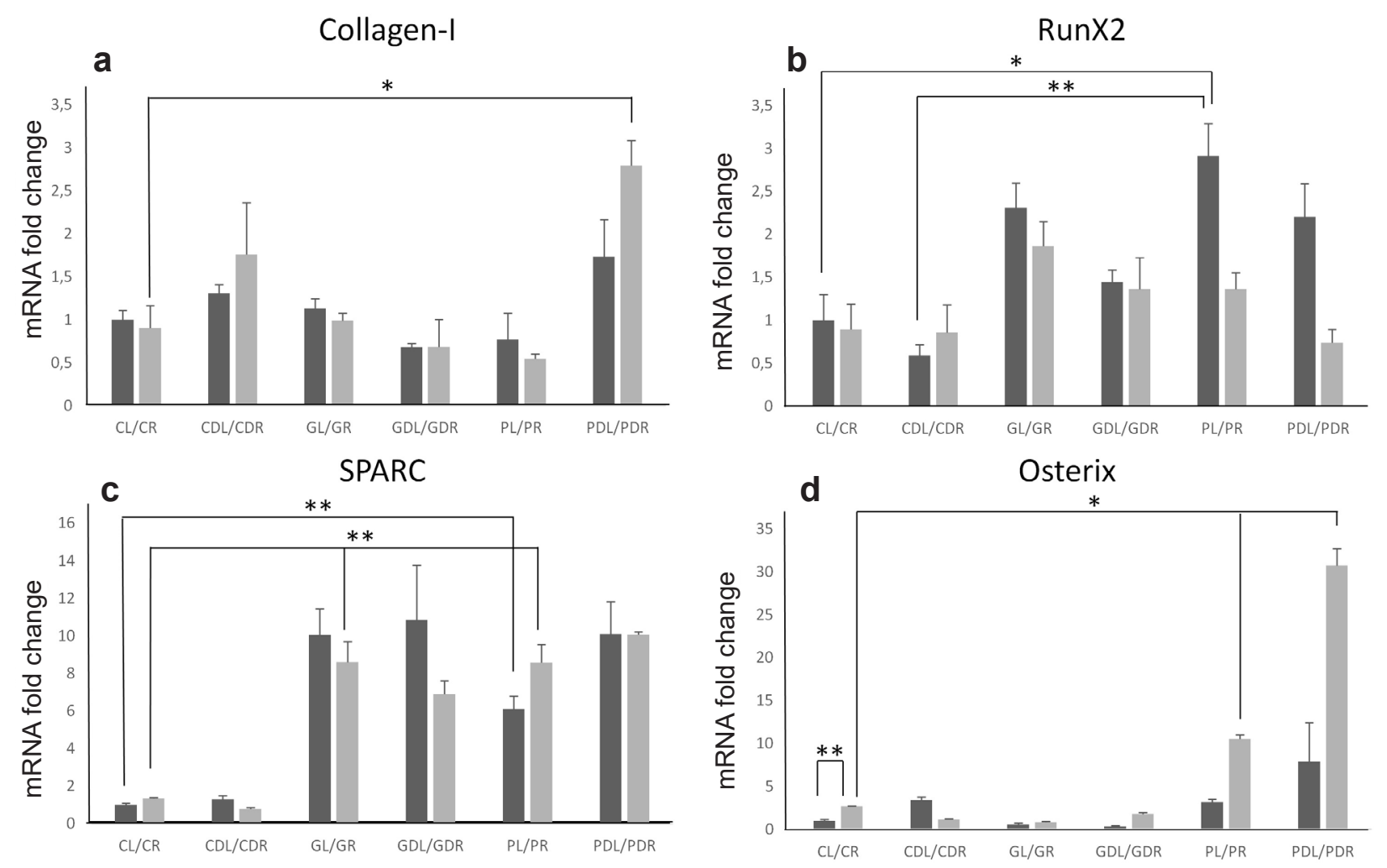

Fig. 7. Osteo-differentiation gene expression of cultured hDPSCs by qPCR. Normalised mRNA expression of hDPSC cultures (14 d) for COL-I, RUNX2, SPARC and OSTERIX analysed by QPCR. Statistical significance $\left({ }^{*} p \leq 0.05\right)$ and $\left({ }^{* *} p \leq 0.01\right)$. CL: control Ti6aI4V; CR: control BAS; CDL: control/ Ti6aI4V/differentiation treatment; CDR: control/BAS/differentiation treatment; GL: Ti6aI4V/PRGF; GR: BAS/PRGF; GDL: Ti6aI4V/ PRGF/differentiation treatment; GDR: BAS/PRGF/differentiation treatment; PL: TI6AI4V/PRF; PR: BAS/PRF; PDL: TI6AI4V/PRF/differentiation treatment; PDR: BAS/PRF/differentiation treatment.

could be identified on the material surface, even at the macroscopic level and in control conditions (Fig. 5b-e). Thus, the mere presence of a titanium surface induced hDPSCs to differentiate towards the osteoblastic phenotype. Pharmacological induction of osteoblastic differentiation only had a modest effect on the absolute number of calcified nodules, with respect to controls, when hDPSCs were seeded upon titanium. However, those calcified accumulations appeared to stain more intensely with alizarin red in hDPSC s subjected to osteoinduction, especially when cells were seeded over BAS surfaces (Fig. 5e,j). This increase in ARS between control and differentiation conditions was far more evident for hDPSCs grown on plastic dishes. In this case, hDPSCs depended highly on the presence of dexamethasone, ascorbic acid and $\beta$-glycerophosphate to osteodifferentiate and be eventually able to generate a calcified extracellular alizarin-red-positive bone-like matrix (Fig. 5a,f).

PRGF and PRF supplementation affect the osteoblastic differentiation of hDPSCs

After assessing that hDPSCs could differentiate into bone-producing osteoblastic cells on different titanium surfaces, the effect of adding PRGF/PRF upon hDPSC osteogenic capability was evaluated. To this end, PRGF and PRF supplements were added to hDPSCs in osteoinduction condition cultured on BAS titanium discs. ARS assays were performed after $14 \mathrm{~d}$ in culture. hDPSC osteodifferentiation and mineralised matrix deposition was found under all conditions, in the presence of either PRGF or PRF. However, it was noteworthy that the presence of PRGF did actually decrease bone production by hDPSCs with respect to control conditions, as assessed by ARS precipitate formation (Fig. 6). In contrast, in the presence of PRF the generated ARS precipitates, despite being apparently no more numerous than in control conditions, did show a particularly intense staining, as shown by photometric quantification (Fig. 6d).

Analysis of osteoblastic differentiation markers by RT-PCR on hDPSCs seeded on Ti6A14V and $B A S^{T M}$ titanium surfaces in the presence or absence of PRGF/PRF

Alizarin red positive assays revealed that the addition of PRGF and PRF to cultures of hDPSC s on Ti6A14V and BAS surfaces was somehow affecting the process of osteoblastic differentiation and mineralisation. However, more information was needed with regard to which particular event(s) could be affected within this process. To asses this question, a battery of gene markers were chosen as being representative of the different stages of osteoblast maturation: 
1. RUNX2, a marker of immature osteoblasts

2. SPARC, a marker of intermediate secretory osteoblasts

3. OSTERIX/SP7, a marker of fully mature mineralising osteoblasts.

It was found that either alone or in the presence of reagents for osteoblast differentiation, hDPSCs cultured on both types of titanium samples expressed COL-I, RUNX2, OSTERIX and SPARC markers at different levels, as assessed by Q-PCR (Fig. 7). In addition, in the presence of PRGF or PRF, the aforementioned markers showed increased levels of expression in most, but not all, conditions. $C O L-I$ expression showed some variability in between control and plasma product-containing samples. However, the expression of pre-osteoblastic commitment markers RUNX2 and SPARC expression significantly increased in the presence of PRGF and PRF. This was especially noticeable in the case of SPARC, whose expression levels consistently rose 6-11 fold when hDPSCs were cultured in the presence of both PRGF and PRF, with respect to standard culture conditions, either with or without osteoblastic differentiation treatment (Fig. 7c). When assessing the effect of PRF and PRGF on osteoblastic differentiation, some interesting differences were found. Despite the increase of preosteoblastic markers RUNX2 and SPARC in the presence of both PRGF and PRF, a clear induction of the fully mature osteoblastic marker OSTERIX was only detected in the presence of PRF, which significantly increased expression levels of this marker 10-30 fold, either in the absence or the presence of the differentiation treatment (Fig. 7d). However, it was noteworthy that the presence of $20 \%$ soluble PRGF did not induce any increase in OSTERIX expression whatsoever, and hDPSCs even showed a lower expression level of this marker than in control conditions.

\section{Discussion}

In the present work, the aim was to evaluate the efficacy of hDPSCs, in combination with autologous plasma components, as an in vitro cell system for bone generation on biomimetic titanium dental implant materials. In this context, the combination of hDPSCs stimulated by PRGF or PRF and cultured on standard Ti6A14V and biomimetic BAS ${ }^{\mathrm{TM}}$ (Avinent Implant System) titanium surfaces were studied in order to test for possible enhancements in the osteoblastic differentiation process out of human mesenchymal cells, as well as their bone-matrix secretion on the implant surface. Since Gronthos et al. isolated hDPSCs for the first time (Gronthos et al., 2000), many laboratories have demonstrated the ability of those ecto-mesenchymal stem cells to differentiate into very different cell lineages (Nuti et al., 2016), including bone-producing osteoblastic cells, whose generation is of utmost interest in the field of implantology (Giuliani et al., 2013; Tatullo, 2017).
The differentiation of mesenchymal stem cells into functional mineralising osteoblasts and osteocytes is a process consisting of at least 5 stages, each of which characterised by the expression of specific gene markers. First, mesenchymal stem cells commit to osteo-chondroprogenitor cells, which start to express the transcription factor RUNX2, which then commits cells to turn into bone-lineage osteoprogenitor cells. At this second stage, cells transform into secretory osteoblasts that produce and secrete calcium-binding proteins SPARC (osteonectin) and BGLAP (osteocalcin), together with Collagen I, to form the organic (osteoid) part of the bone extracellular matrix. Later on, this immature bone matrix becomes mineralised by hydroxyapatite mineral deposition, a process which requires the crystal nucleation and growth by reaction of $\mathrm{Ca}^{2+}$ and $\mathrm{PO}^{4-}$ ions, which is catalysed by ALP activity. By the time cells become mineralising they also express markers of fully mature differentiated osteoblasts, such as the transcription factor OSTERIX. This marker is also retained in mature osteocytes, once osteoblasts are surrounded by the mature calcified bone matrix. Mature osteocytes within lacunae also keep expressing, albeit at lower levels, SPARC (osteonectin) and Collagen I, together with other bone matrix proteins, to participate in bone matrix remodelling throughout time (Fig. 8).

Changes to the composition and the surface of titanium implants are generating important improvements to the biocompatibility of these materials for clinical use, especially with a view to activating osteoblast differentiation and bone deposition around the titanium pillar. In this regard, previous work has demonstrated the ability of human mesenchymal stem cells to adhere, proliferate and differentiate to osteoblasts when seeded on titanium surfaces, even without the addition of any osteogenic differentiation factor to the cell culture medium (Olivares-Navarrete et al., 2010). The results of the present work corroborate those previous reports, showing that mesenchymal phenotype cells - in this case hDPSCs derived from the same donor patient - can behave very differently depending on the substrate they are growing on. The presence of a titanium attachment surface on its own was able to induce differentiation of hDPSCs to mature osteoblasts that could produce a mineralised bone matrix stainable with alizarin red. This came in contrast to what is usually found when standard plastic culture surfaces are used, where osteoblastic differentiation does not occur unless the stem cell cultures are treated for several days with dexamethasone, $\beta$-glycerophosphate, and ascorbate (Langenbach and Handschel, 2013). One of the most important advantages of titanium surfaces is the stimulation of mesenchymal stem cell differentiation to osteoblasts. It has been reported that both the macro and microroughness of the titanium surface also play an important role in osteoblast attachment, proliferation and differentiation (Boyan 
et al., 2016; Coelho et al., 2009). When hDPSCs are seeded on porous surfaces they show osteoblast differentiation, production of appreciable amounts of bone morphogenetic proteins as well as vascular endothelial growth factor and specific bone proteins (Perrotti et al., 2013). Recent work suggests that chemically modified microrough titanium surfaces are more osteoinductive, which represents a significant advantage for application in the dental clinic (Boyan et al., 2018; DE Colli et al., 2018). In the present study a small but significant difference in the levels of expression of OSTERIX was found, when comparing Ti6Al4V (smooth) and BAS (rough biomimetic) surface-grown hDPSCs in control (no osteoblastic stimulation) conditions. However, the osteoinductive effect on BAS surfaces could be greatly enhanced by the presence of platelet rich fibrin (PRF), either in combination or not with osteoblastic differentiation treatments. Under these conditions the OSTERIX mRNA expression was raised by more than one order of magnitude.

Both Ti6Al4V (smooth) and BAS (rough) implant surfaces were also tested for the in vitro maintenance and expansion of hDPSCs. It was found that both surfaces preserved cell viability and allowed a good cell proliferation, as assessed by the detection of large numbers of proliferative Ki67 positive cells in basal conditions. Despite both plasma formulations being permissive for the growth of hDPSCs, cell proliferation was significantly enhanced by the presence of $20 \%$ soluble PRGF, but not by insoluble PRF. Perhaps this interesting difference could be related to the particular application method of both plasma products. In the case of PRGF, the soluble plasma fraction was applied at a proportion of $20 \%$, and renewed with every medium change every 2-3 d. In contrast, PRF membranes were introduced into the culture wells and maintained there over the period

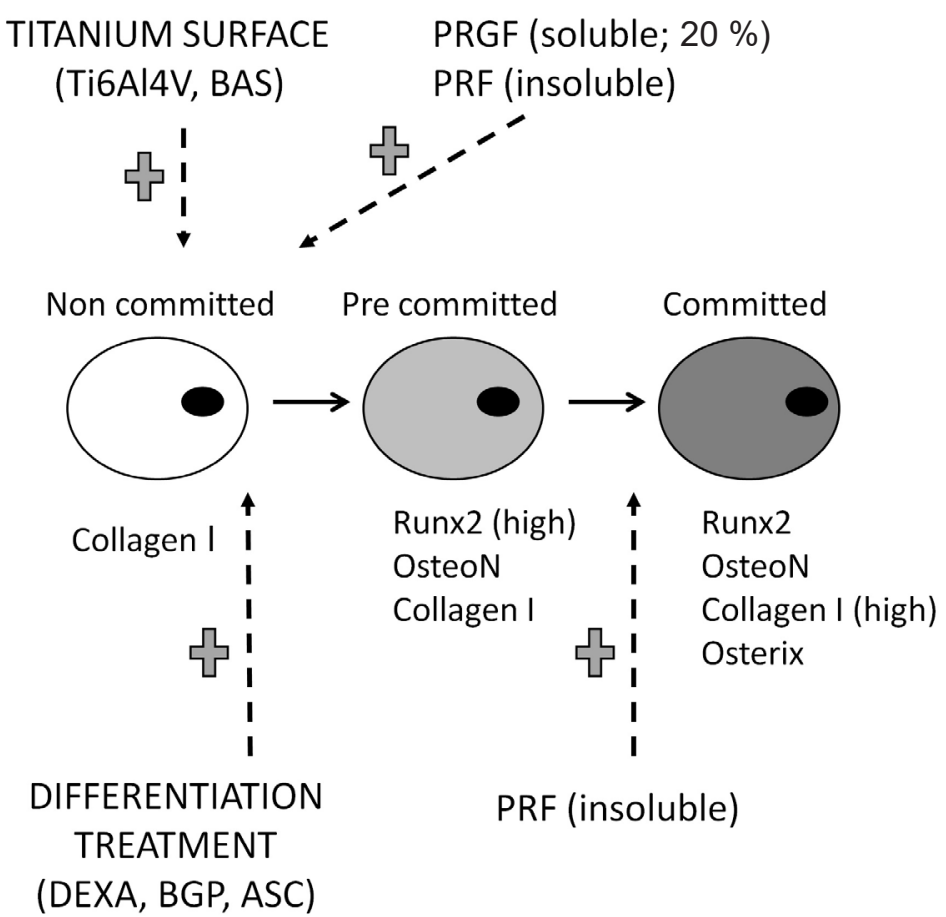

Fig. 8. Summary of plasma derived product effects on osteo-differentiation process of hDPSC cultures. Theoretical model summarising the different effects elicited by the growth substrate and/or supplementation with pharmacological differentiation reagents and plasma derived products (PRGF/PRF) over the osteoblastic differentiation process of hDPSCs. Mesenchymal stem cells grow in an undifferentiated state in vitro, and can be induced to commitment to osteoblastic differentiation, where in an initial stage cells express the transcription factor RUNX2, as well as progressively higher amounts of osteoid components like Collagen-I, and calcium-binding proteins (SPARC/Osteonectin). In a terminal stage of osteoblastic differentiation, the cells acquire the capacity to mineralise the osteoid matrix, to form a mature bone tissue. This is characterised at the cellular/molecular level by the rise of expression of the mature osteoblast/osteocyte gene marker OSTERIX. Titanium surface porosity, pharmacological reagents as well as plasma-derived PRGF and PRF appear all to influence the mesenchymal stem cell to preosteoblast transition, as higher levels of RUNX2 and SPARC expression and/or ALP staining are found in all these conditions. However, hDPSCs grown in the permanent presence of PRGF in soluble formulation do not manage to pass beyond the preosteoblastic stage, possibly because of conflictive proliferative/differentiation signaling. In contrast, hDPSCs grown in the presence of PRF membranes present the highest levels of osteoblastic differentiation, especially when the cells are seeded on biomimetic BAS titanium surfaces combined with osteoblastic differentiation reagents. 
of the experiments. It is plausible to hypothesise that a high concentration of growth factors that induced hDPSC proliferation, as it happened in the case of soluble PRGF supplementation, would paradoxically have a negative effect over terminal osteoblastic differentiation, as both processes (cell proliferation vs. differentiation) are mutually exclusive and antagonistic to each other. In PRGF conditions, an increase of osteoblastic pre-commitment was actually found, as assessed by an increase of RUNX2 and SPARC mRNA levels, but somehow hDPSCs failed to terminally differentiate to mature osteoblasts, as shown by a reduction in OSTERIX expression and ARS. In contrast, supplementation of hDPSC cultures with insoluble PRF membranes would arguably generate conditions whereby a slow gradual release of growth factors from the fibrin clot would eventually favour terminal differentiation of hDPSCs to osteoblastic cells. It is noteworthy that the combination of biomimetic BAS surfaces with PRF was apparently the most effective condition to induce in vitro osteogenesis by hDPSCs on titanium surfaces. This is where the presence of a rough BAS titanium surface proved to be most valuable with respect to the smooth Ti6Al4V one, as OSTERIX expression levels could be enhanced very significantly when BAS surfaces were combined with PRF.

Finally, more in vivo studies will be necessary to achieve a full translation of this methodology to the dental clinic. Despite being not the most abundant source of mesenchymal stem cells in the human body, hDPSCs from the dental pulp are of particular interest for the fields of implantology and craniomaxillofacial surgery. This holds especially true for the cases of patients with oral and maxillofacial trauma who lose several teeth in an accidental episode. They might very well benefit from an approach whereby clinicians took advantage of the lost dental pieces to extract autologous hDPSCs to aid in the reconstruction of the whole affected bone area. Whatever the scenario, these cells respond very effectively to osteoblastic differentiation protocols when grown on microporous biomimetic titanium surfaces such as BAS ${ }^{\mathrm{TM}}$, especially in combination with non-soluble fibrin-clot based human plasma products.

\section{Conclusions}

The results obtained for this in vitro model of osteoblastic differentiation out of hDPSCs suggests a combination of biomimetic rough titanium surfaces (BAS ${ }^{\mathrm{TM}}$ ) with autologous plasma-derived fibrin-clot membranes, such as PRF and/or PRGF formulations, to maximise osteoblastic cell differentiation out of mesenchymal stem cells, the generation of calcified bone matrix, and the osteo-integration of titaniummade dental implants. By means of different gene and biochemical markers, the osteoblastic differentiation process was dissected to its different steps to validate the best conditions to obtain populations of fully differentiated bone-producing cells upon titanium surfaces. These findings provide strong experimental support to the common clinical practice of applying plasma-derived fibrin clot membranes to increase bone deposition around microporous titanium implant surfaces, thereby improving their anchorage.

\section{Acknowledgements}

This research was supported by the University of the Basque Country (UPV/EHU; GIU16/66), the Basque Government (Elkartek KK-2019/00093) and Avinent Implant System S.L.

Technical and human support from Dr. R. Andrade and Dr. A. Díez-Torre in the SGIKER high resolution microscopy facility of UPV/EHU is also greatly acknowledged.

\section{Conflict of interests}

The authors certify that they have no affiliations with or involvement in any organisation or entity with any financial interest in the subject matter or materials discussed in this manuscript.

\section{References}

Albrektsson T, Brånemark PI, Hansson HA, Lindström J (1981) Osseointegrated titanium implants. Requirements for ensuring a long-lasting, direct bone-to-implant anchorage in man. Acta Orthop Scand 52: 155-170.

Anitua E, Alkhraisat MH, Orive G (2012) Perspectives and challenges in regenerative medicine using plasma rich in growth factors. J Control Release 157: 29-38.

Anitua E, Orive G, Pla R, Roman P, Serrano V, Andía I (2009) The effects of PRGF on bone regeneration and on titanium implant osseointegration in goats: a histologic and histomorphometric study. J Biomed Mater Res A 91: 158-165.

Anitua E, Tejero R, Zalduendo MM, Orive G (2013) Plasma rich in growth factors promotes bone tissue regeneration by stimulating proliferation, migration, and autocrine secretion in primary human osteoblasts. J Periodontol 84: 1180-1190.

Anitua E, Troya M, Zalduendo M, Tejero R, Orive G (2016) Progress in the use of autologous regenerative platelet-based therapies in implant dentistry. Curr Pharm Biotechnol 17: 402-413.

Annunziata M, Guida L (2015) The effect of titanium surface modifications on dental implant osseointegration. Front Oral Biol 17: 62-77.

Aurrekoetxea M, Garcia-Gallastegui P, Irastorza I, Luzuriaga J, Uribe-Etxebarria V, Unda F, Ibarretxe G (2015) Dental pulp stem cells as a multifaceted tool for bioengineering and the regeneration of craniomaxillofacial tissues. Front Physiol 6: 289. 
Boyan BD, Cheng A, Olivares-Navarrete R, Schwartz Z (2016) Implant surface design regulates mesenchymal stem cell differentiation and maturation. Adv Dent Res 28: 10-17.

Boyan BD, Olivares-Navarrete R, Berger MB, Hyzy SL, Schwartz Z (2018) Role of Wnt11 during osteogenic differentiation of human mesenchymal stem cells on microstructured titanium surfaces. Sci Rep 8: 8588.

Coelho PG, Granjeiro JM, Romanos GE, Suzuki M, Silva NRF, Cardaropoli G, Thompson VP, Lemons JE (2009) Basic research methods and current trends of dental implant surfaces. J Biomed Mater Res Part B Appl Biomater 88: 579-596.

DE Colli M, Radunovic M, Zizzari VL, DI Giacomo V, DI Nisio C, Piattelli A, Calvo Guirado JL, Zavan B, Cataldi A, Zara S (2018) Osteoblastic differentiating potential of dental pulp stem cells in vitro cultured on a chemically modified microrough titanium surface. Dent Mater J 37: 197-205.

Gasik M, Braem A, Chaudhari A, Duyck J, Vleugels J (2015) Titanium implants with modified surfaces: meta-analysis of in vivo osteointegration. Mater Sci Eng C Mater Biol Appl 49: 152-158.

Giannini S, Cielo A, Bonanome L, Rastelli C, Derla C, Corpaci F, Falisi G (2015) Comparison between PRP, PRGF and PRF: lights and shadows in three similar but different protocols. Eur Rev Med Pharmacol Sci 19: 927-930.

Giuliani A, Manescu A, Langer M, Rustichelli F, Desiderio V, Paino F, De Rosa A, Laino L, d'Aquino R, Tirino V, Papaccio G (2013) Three years after transplants in human mandibles, histological and in-line holotomography revealed that stem cells regenerated a compact rather than a spongy bone: biological and clinical implications. Stem Cells Transl Med 2: 316-324.

Graziano A, d'Aquino R, Cusella-De Angelis MG, De Francesco F, Giordano A, Laino G, Piattelli A, Traini T, De Rosa A, Papaccio G (2008a) Scaffold's surface geometry significantly affects human stem cell bone tissue engineering. J Cell Physiol 214: 166172.

Graziano A, d'Aquino R, Laino G, Papaccio G (2008b) Dental pulp stem cells: a promising tool for bone regeneration. Stem Cell Rev 4: 21-26.

Gronthos S, Mankani M, Brahim J, Robey PG, Shi S (2000) Postnatal human dental pulp stem cells (DPSCs) in vitro and in vivo. Proc Natl Acad Sci U S A 97: 13625-13630.

Ito K, Yamada Y, Nakamura S, Ueda M (2011) Osteogenic potential of effective bone engineering using dental pulp stem cells, bone marrow stem cells, and periosteal cells for osseointegration of dental implants. Int J Oral Maxillofac Implants 26: 947-954.

Jemat A, Ghazali MJ, Razali M, Otsuka Y (2015) Surface modifications and their effects on titanium dental implants. Biomed Res Int 2015: 791725.

Jovani-Sancho MDM, Sheth CC, Marqués-Mateo M, Puche-Torres M (2016) Platelet-rich plasma: a study of the variables that may influence its effect on bone regeneration. Clin Implant Dent Relat Res 18: 1051-1064.

Karbanová J, Soukup T, Suchánek J, Mokrý J (2010) Osteogenic differentiation of human dental pulp-derived stem cells under various ex-vivo culture conditions. Acta Medica (Hradec Kralove) 53: 79-84.

Khurana R, Kudva PB, Husain SY (2017) Comparative evaluation of the isolation and quantification of stem cells derived from dental pulp and periodontal ligament of a permanent tooth and to assess their viability and proliferation on a plateletrich fibrin scaffold. J Indian Soc Periodontol 21: 16-20.

Kobayashi E, Flückiger L, Fujioka-Kobayashi M, Sawada K, Sculean A, Schaller B, Miron RJ (2016) Comparative release of growth factors from PRP, PRF, and advanced-PRF. Clin Oral Investig 20: 23532360 .

Kumar KR, Genmorgan K, Abdul Rahman SM, Rajan MA, Kumar TA, Prasad VS (2016) Role of plasma-rich fibrin in oral surgery. J Pharm Bioallied Sci 8: S36-S38.

La Noce M, Mele L, Tirino V, Paino F, De Rosa A, Naddeo P, Papagerakis P, Papaccio G, Desiderio V (2014a) Neural crest stem cell population in craniomaxillofacial development and tissue repair. Eur Cell Mater 28: 348-357.

La Noce M, Paino F, Spina A, Naddeo P, Montella R, Desiderio V, De Rosa A, Papaccio G, Tirino V, Laino L (2014b) Dental pulp stem cells: state of the art and suggestions for a true translation of research into therapy. J Dent 42: 761-768.

Laino G, Carinci F, Graziano A, d'Aquino R, Lanza V, De Rosa A, Gombos F, Caruso F, Guida L, Rullo R, Menditti D, Papaccio G (2006) In vitro bone production using stem cells derived from human dental pulp. J Craniofac Surg 17: 511-515.

Langenbach F, Handschel J (2013) Effects of dexamethasone, ascorbic acid and $\beta$-glycerophosphate on the osteogenic differentiation of stem cells in vitro. Stem Cell Res Ther 4: 117.

Le Guéhennec L, Soueidan A, Layrolle P, Amouriq Y (2007) Surface treatments of titanium dental implants for rapid osseointegration. Dent Mater 23: 844-854.

Mangano C, De Rosa A, Desiderio V, d'Aquino R, Piattelli A, De Francesco F, Tirino V, Mangano F, Papaccio G (2010) The osteoblastic differentiation of dental pulp stem cells and bone formation on different titanium surface textures. Biomaterials 31: 3543-3551.

Masuki H, Okudera T, Watanebe T, Suzuki M, Nishiyama K, Okudera H, Nakata K, Uematsu K, Su C-Y, Kawase T (2016) Growth factor and pro-inflammatory cytokine contents in plateletrich plasma (PRP), plasma rich in growth factors (PRGF), advanced platelet-rich fibrin (A-PRF), and concentrated growth factors (CGF). Int J Implant Dent 2: 19.

Mortada I, Mortada R (2018) Dental pulp stem cells and osteogenesis: an update. Cytotechnology 70: 1479-1486. 
Naves MM, Menezes HHM, Magalhães D, Ferreira JA, Ribeiro SF, de Mello JDB, Costa HL (2015) Effect of macrogeometry on the surface topography of dental implants. Int J Oral Maxillofac Implants 30: 789-799.

Nishiyama K, Okudera T, Watanabe T, Isobe K, Suzuki M, Masuki H, Okudera H, Uematsu K, Nakata K, Kawase T (2016) Basic characteristics of plasma rich in growth factors (PRGF): blood cell components and biological effects. Clin Exp Dent Res 2: 96-103.

Nuti N, Corallo C, Chan BMF, Ferrari M, GeramiNaini B (2016) Multipotent differentiation of human dental pulp stem cells: a literature review. Stem Cell Rev 12: 511-523.

Olivares-Navarrete R, Hyzy SL, Hutton DL, Erdman CP, Wieland M, Boyan BD, Schwartz Z (2010) Direct and indirect effects of microstructured titanium substrates on the induction of mesenchymal stem cell differentiation towards the osteoblast lineage. Biomaterials 31: 2728-2735.

Paino F, La Noce M, Giuliani A, De Rosa A, Mazzoni S, Laino L, Aer E, Papaccio G, Desiderio V, Tirino V (2017) Human DPSCs fabricate vascularized woven bone tissue: a new tool in bone tissue engineering. Clin. Sci. 131: 699-713.

Paknejad M, Shayesteh YS, Yaghobee S, Shariat S, Dehghan M, Motahari P (2012) Evaluation of the effect of plasma rich in growth factors (PRGF) on bone regeneration. J Dent (Tehran) 9: 59-67.

Perrotti V, Palmieri A, Pellati A, Degidi M, Ricci L, Piattelli A, Carinci F (2013) Effect of titanium surface topographies on human bone marrow stem cells differentiation in vitro. Odontology 101: 133-139.

Rani VVD, Vinoth-Kumar L, Anitha VC, Manzoor K, Deepthy M, Shantikumar VN (2012) Osteointegration of titanium implant is sensitive to specific nanostructure morphology. Acta Biomater 8: 1976-1989.

Rupp F, Liang L, Geis-Gerstorfer J, Scheideler L, Hüttig F (2018) Surface characteristics of dental implants: A review. Dent Mater 34: 40-57.

Salou L, Hoornaert A, Stanovici J, Briand S, Louarn G, Layrolle P (2015) Comparative bone tissue integration of nanostructured and microroughened dental implants. Nanomedicine (Lond) 10: 741-751.

Santander S, Alcaine C, Lyahyai J, Pérez MA, Rodellar C, Doblaré M, Ochoa I (2012) In vitro osteoinduction of human mesenchymal stem cells in biomimetic surface modified titanium alloy implants. Dent Mater J 31: 843-850.

Tatullo M ed. (2017) MSCs and Innovative Biomaterials in Dentistry. Humana Press. Stem Cell Biology and Regenerative Medicine. 188pp ISBN 978-3-319-55644-4

\section{Discussion with Reviewer}

Giovanna Orsini: Is there evidence for an effect of implant surface modifications on other phenomena aside from direct osteoblastic differentiation, e.g. angiogenesis?

Authors: We have not assessed the production of angiogenic factors in this study, although there is important evidence that hDPSCs can also differentiate to endothelial cells ( $d$ 'Aquino et al., 2007; Luzuriaga et al., 2019, additional references). It remains to be characterised whether endothelial differentiation of hDPSCs may also play a role in providing vascular support to improve the osteointegration of titanium implants within the alveolar bone.

\section{Additional References}

d'Aquino R, Graziano A, Sampaolesi M, Laino G, Pirozzi G, De Rosa A, Papaccio G (2007) Human postnatal dental pulp cells co-differentiate into osteoblasts and endotheliocytes: a pivotal synergy leading to adult bone tissue formation. Cell Death Differ 14: 1162-1171.

Luzuriaga J, Pastor-Alonso O, Encinas JM, Unda F, Ibarretxe G, Pineda JR (2019) Human dental pulp stem cells grown in neurogenic media differentiate into endothelial cells and promote neovasculogenesis in the mouse brain. Front Physiol 10: 347.

Editor's note: The Scientific Editor responsible for this paper was Thimios Mitsiadis. 